\title{
Comparison of PID Controller with Model Predictive Controller for Milk Pasteurization Process
}

\author{
Tesfaye Alamirew $^{\star 1}$, V. Balaji ${ }^{2}$, Nigus Gabbeye ${ }^{3}$ \\ ${ }^{1,3}$ Faculty of Chemical and Food Engineering, Bahir Dar University, Ethiopia \\ ${ }^{2}$ Faculty of Electrical Engineering, Bahir Dar University, Ethiopia \\ *Corresponding author, e-mail: jaliniezeco@hotmail.com
}

\begin{abstract}
Proportional-Integral-Derivative (PID) controllers are used in many of the Industries for various process control applications. PID controller yields a long settling time and overshoot which is not good for the process control applications. PID is not suitable for many of the complex process control applications. This research paper is about developing a better type of controller, known as MPC (Model Predictive Control). The aim of the paper is to design MPC and PID for a pasteurization process. In this manuscript comparison of PID controller with MPC is made and the responses are presented. MPC is an advanced control strategy that uses the internal dynamic model of the process and a history of past control moves and a combination of many different technologies to predict the future plant output. The dynamics of the pasteurization process was estimated by using system identification from the experimental data. The quality of different model structures was checked using best fit with data validation, residual and stability analysis. Auto-regressive with exogenous input (ARX322) model was chosen as a model structure of the pasteurization process and fits about $80.37 \%$ with datavalidation. MPC and PID control strategies were designed using ARX322 model structure. The controller performance was compared based on settling time, percent of overshoot and stability analysis and the results are presented.
\end{abstract}

Keywords: pasteurization process, PID, MPC, ARX model, ARMAX model, BJ model, OE model

\section{Introduction}

In a modern world the economic and quality issues become more and more important, efficient control systems have become indispensable. Therefore the process industries require more reliable, accurate, robust, efficient and flexible control systems for the operation of process plant. In order to fulfill the above requirements there is a continuing need for research on improved forms of control [1].

Control of temperature plays an important role in pasteurization plants. High temperature short time (HTST) is keeping milk or other food stuffs at $720 \mathrm{C}$ for 15 seconds in insulated holding tube. The pasteurization process consists of three stages like regeneration, heating and cooling sections. The crucial stage is heating process using heat exchanger to ensure unpasteurized product achieve desired pasteurization temperature before pass through holding tube and cooling sections. Prior to pasteurize milk sample, the equipment must have adequate controller to control the outlet temperature in order to maintain at standard value [2].

The proportional integral (PI) and proportional integral derivative (PID) controllers are widely used in many industrial control systems because of its simple structure. This controllers are designed without process constraints only use mathematical expression based on error from a set point. In these circumstances, conventional controllers (PI and PID) are no longer to provide adequate and achievable control performance over the whole operating range. Thus designing a controller considering the process constraints and optimize the control performance is essential [3].

Model Predictive Control also known as receding horizon control, is an advanced strategy for optimizing the performance of multivariable control systems. MPC generates control actions by optimizing an objective function repeatedly over a finite moving prediction horizon, within system constraints, and based on a model of the dynamic system to be controlled [4]. 


\section{Process Description}

The plant PCT23, manufactured by Armfield (UK), is a laboratory version of a real industrial pasteurization process, as shown in Figure 1. It consists of a bench-mounted process unit to which is connected a dedicated control console. An interface card DT2811 is used for monitoring and controlling the process through a computer [5].

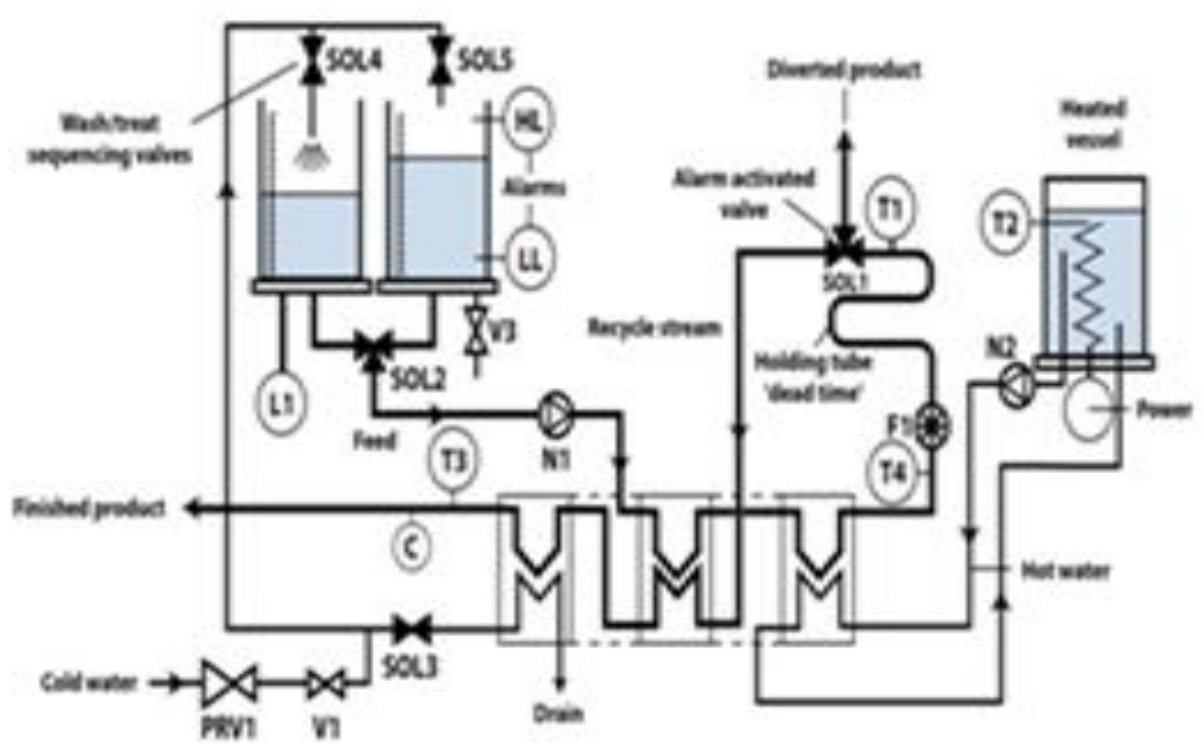

Figure 1. Process flow diagram of pasteurization plant

Here temperature $\mathrm{T} 1$ is the controlled variable and milk flow rate (N1), hot water flow rate (N2) and power are manipulated variables.

\section{Experimental Setup}

\subsection{Input-output Data}

The input-output data was generated by introducing step input in manipulated variables (N1, N2 and power input) and record temperature response (T1). Two input-output data was generated for model estimation and validation as shown in Figure 2.

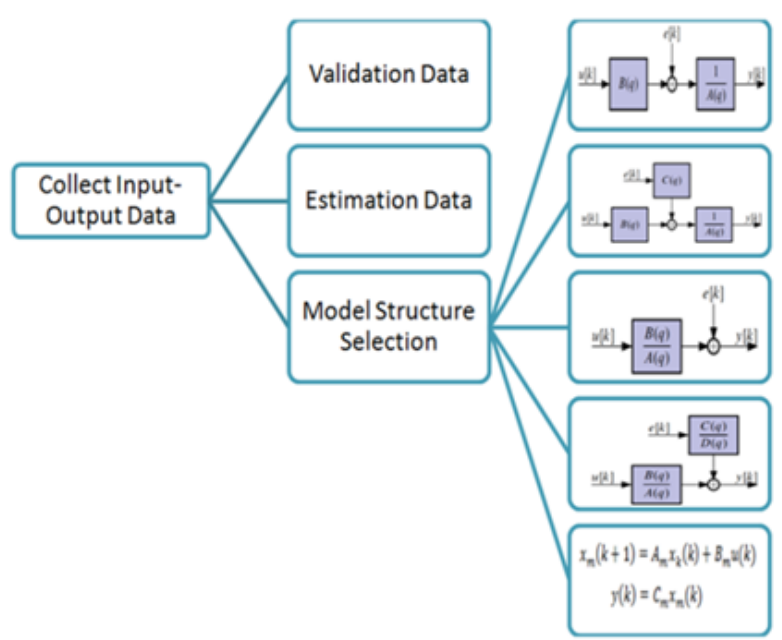

Figure 2. Systematic procedure for model identification process 


\subsection{System identification}

The input-output data was analyzed by the System Identification toolbox in MATLAB. The continuous and discrete model structures were tried to select the model structure that have best fit with validation. Then the selected model structure is tested for residual analysis and pole-zero analysis to check the model stability. The continuous time (CT) model, Autoregressive with exogenous input (ARX) model structures, ARMAX (auto regressive with moving average and exogenous (or extra) input model, Output-Error (OE) model and state space model structures were tried get best model structure in terms of best fit with validation data and model stability for further controller design [6].

Best fit is calculated as:

Validation data $(\mathrm{y})$ is estimated data and is mean of validation data. After selection of best fit model structures model quality analysis like residual and pole zero location should be checked to select a nice and simple model for further controller design. The prediction error or residual is the key quantity. It is defined as the stability of a system can be easily inferred by examining the pole locations of the transfer function [7].

\subsection{Controller Design}

Controllers are basically employed in a closed loop control system. Closed loop control system is one that automatically changes the output based on the difference between the feedback signals to the input signal. Controller is an element used to produce manipulated variable from error variable, for control action [8-9].

a. PID controller

The error signal $e(t)$ is the difference between the reference input $r(t)$ and desired output $y(t)$. This error is manipulated by the PID controller to produce a command signal for the system. PID controller is described by the following transfer function in the continuous sdomain.

b. Model Predictive Control

The model predictive uses quadratic minimization problem. Subject to input and output constraints of the system, where is the set point, Q1 is output weight and Q2 is input weight as shown in Figure 3 . The size of this minimization function and weight matrixes are depend on prediction and control horizon [10-11.]

\section{Results and Discussions}

\subsection{Input-output Data}

Two different experiments are done for model estimation and validation purposes at sampling time of 10 seconds.
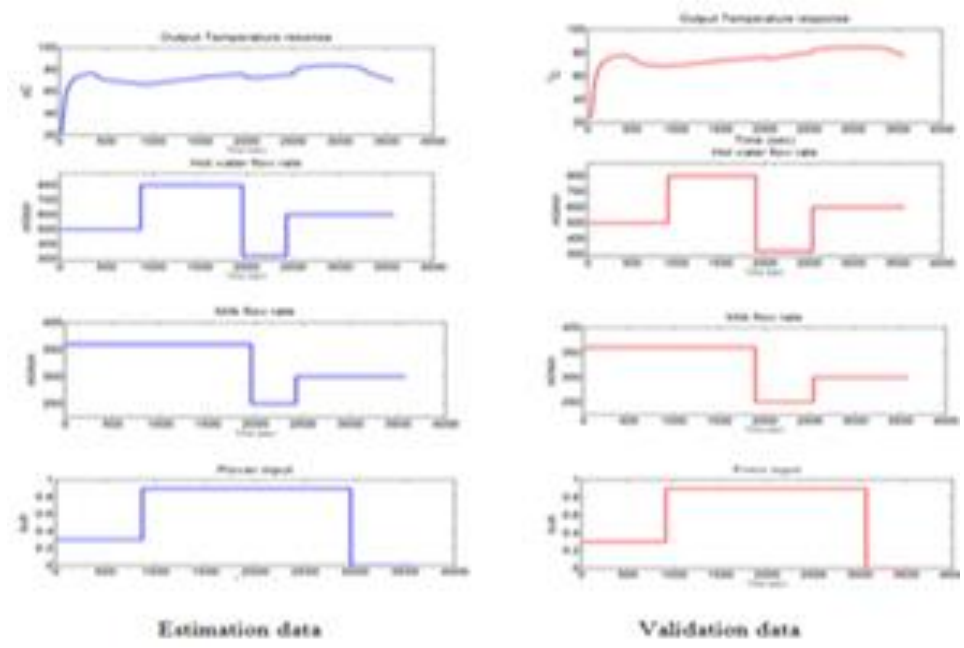

Figure 3. Input-output data profile 


\subsection{Model Structure Selection}

The continuous time model fits the validation data better than the others. ARX422, ARMAX3202 and state space model pss2 are also fits the validation data fairly. The continuous time model doesn't mean a good model rather the selection of model needs further analysis, as shown in Figure 4.

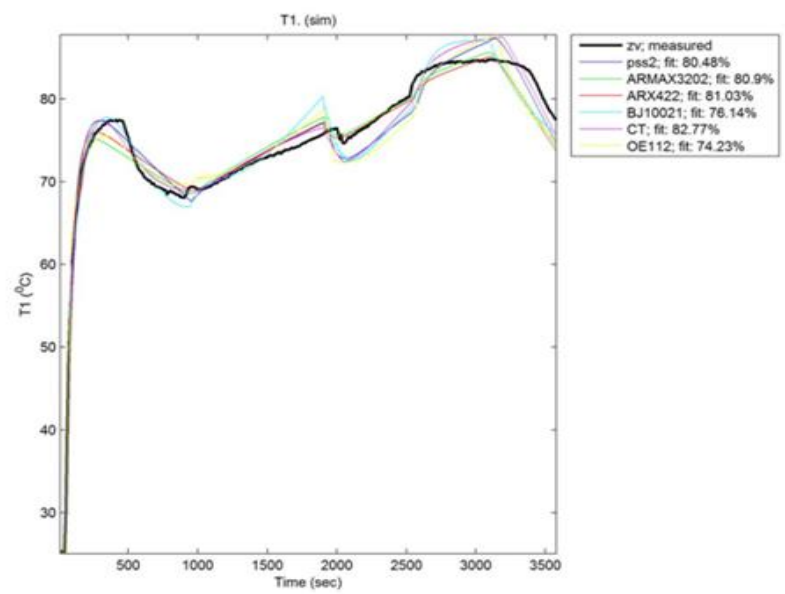

Figure 4. Fits of different model structures

\subsection{Model Quality Analysis}

\subsubsection{Residual Analysis}

For different model structures the auto corelation of residuals for the output (whitness test) and cross correlation of residuals with the input (independence test) were analyzed. From the graphs the horizontal scale is the number of lags, which is the time difference (in samples) between the signals at which the correlation is estimated. The yellow region on the plot represents the confidence interval of the corresponding estimates. Any fluctuations within the confidence interval are considered to be insignificant. A good model should have residuals uncorrelated with past inputs (independence test) and past outputs (whitness test). For poor models either auto and cross corelation residuals or two of residuals is out of the yelllow or confidence regionion as shown in Figure 5.

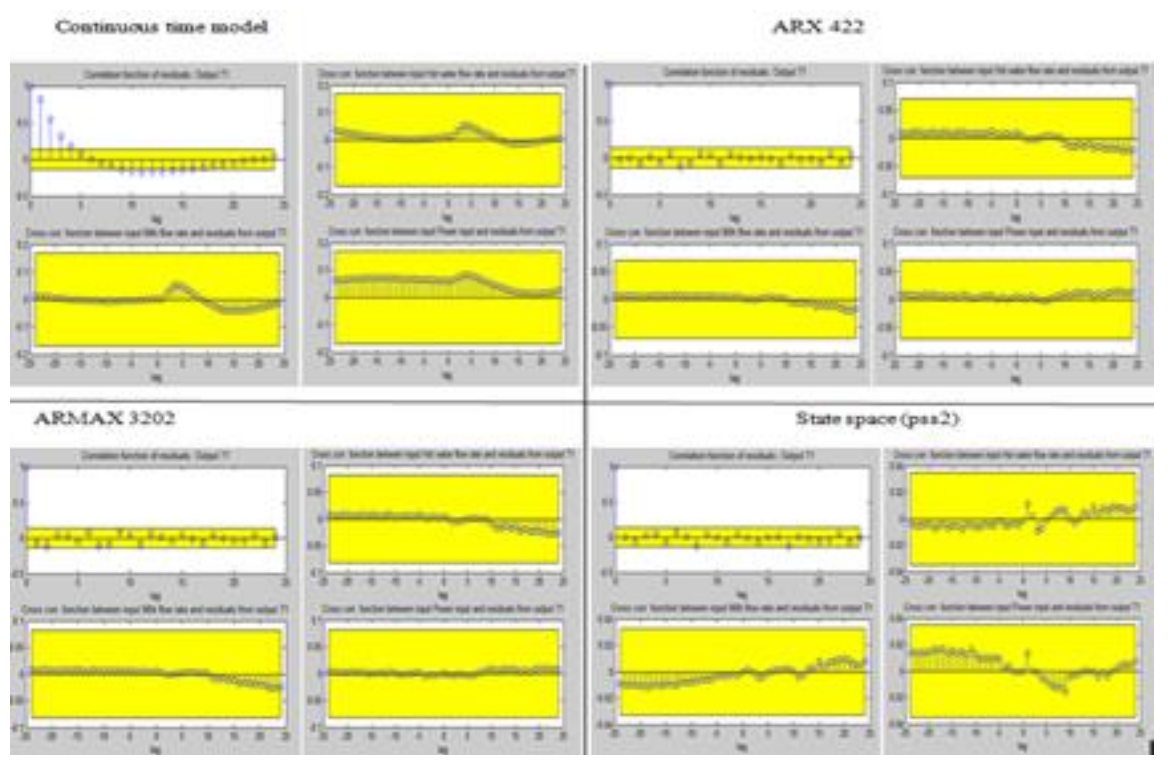

Figure 5. Residual analysis for different model structures 
Only auto correlation analysis of continuous time model analysis shown as some residuals is out of the confidence region. This means this model fail the residual analysis and the other three models still needs other model quality analysis to choose which model is better to represent the pasteurization process.

\subsubsection{Pole-zero location}

Zeros and the poles are equivalent ways of describing the coefficients of a linear difference equation. Poles are associated with the output side of the difference equation, and zeros are associated with the input side of the equation. Generally for stable process all of the poles of discrete time model should be found within unit circle otherwise the model is not stable as shown in Figure 6-8.
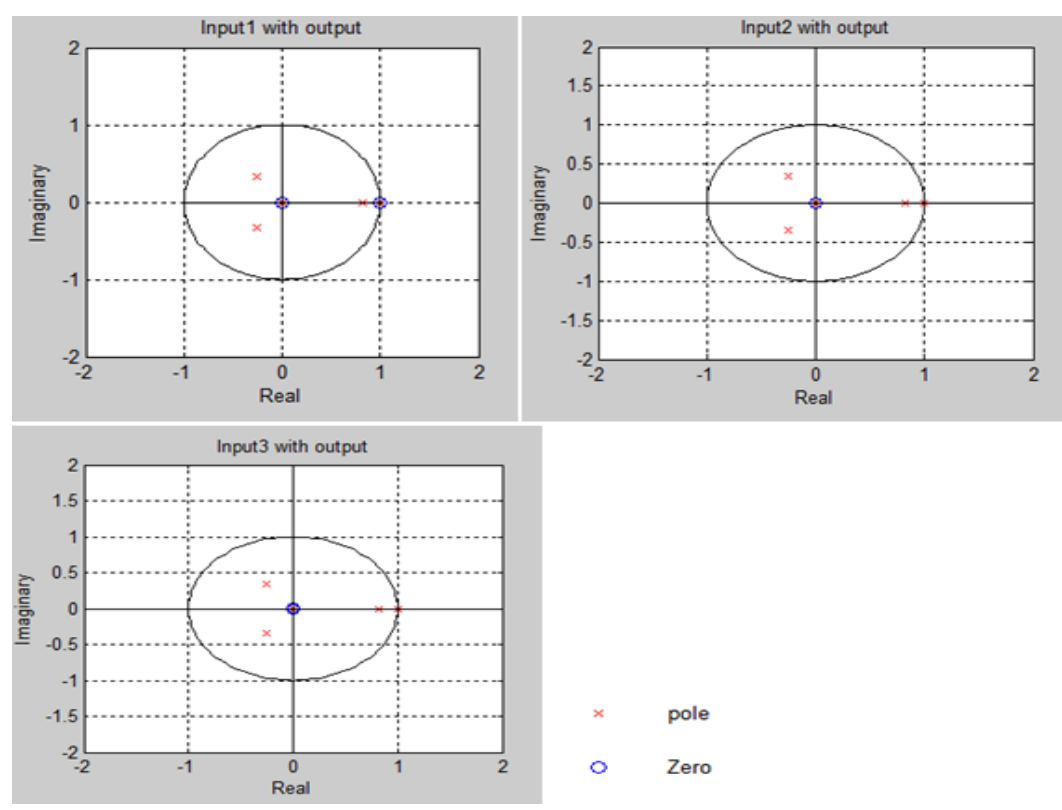

Figure 6. Pole-zero location of ARX422 model
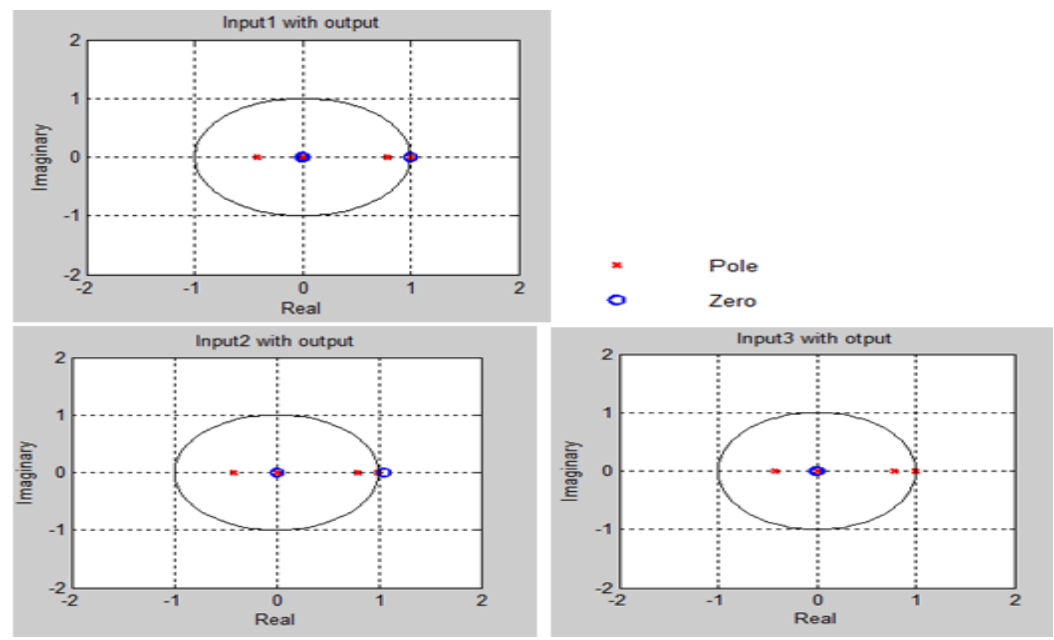

Figure 7. Pole-zero location of ARMAX3202 model 

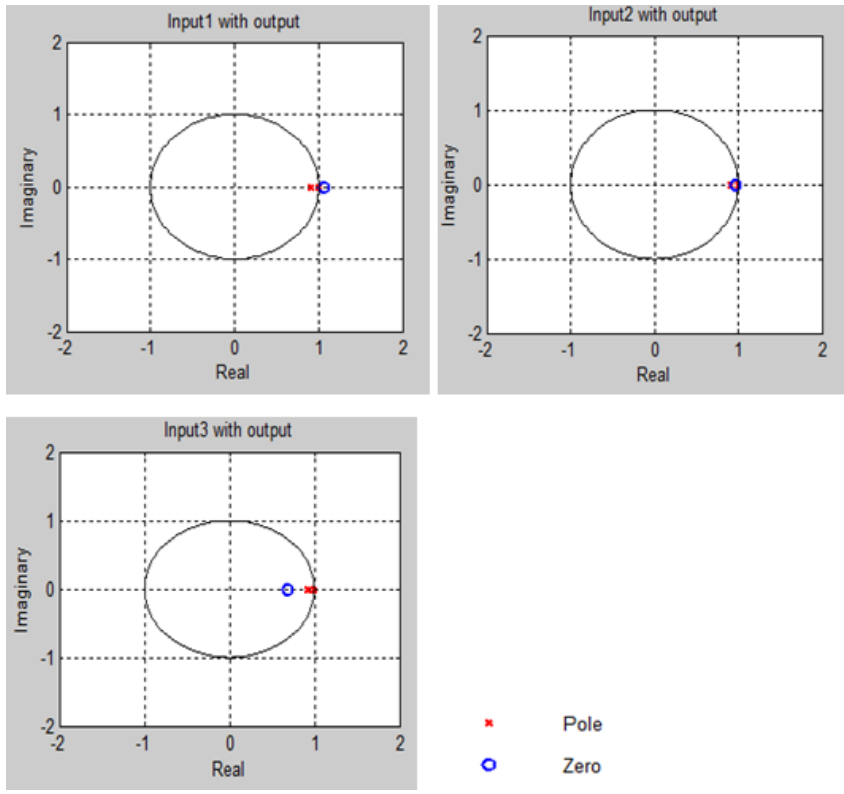

Figure 8. Pole-zero location of state space model

The ARX422 model is stable and there is a chance for pole-zero cancelation. Therefore we can reduce the model order. The ARMAX3202 also stable but the state space model is marginally stable. This model is rejected here.

\subsection{Model Reduction}

The ARX422 reduced to ARX322, still the fit is greater than ARMAX3202. Further reduction below this order deteriorates the fit. The reduced model (Figure 9) also passes the model quality analysis. Therefore the ARX322 model represents the pasteurization process.

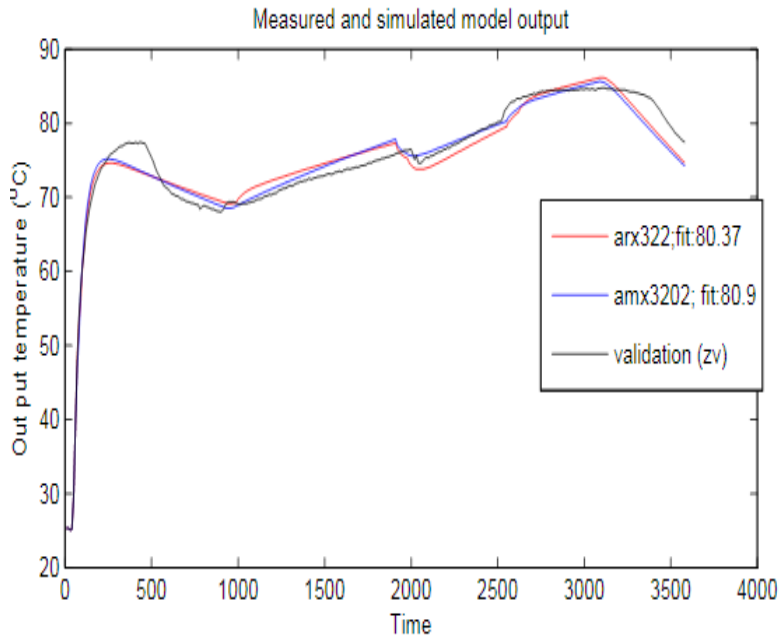

Figure 9. Reduced model fit

The following equation is the converted discrete ARX model to continuous dynamic model for PCT23 pasteurization plant. The process has three inputs that come parallel and one output. The process dynamics is the third order process. 
Input delays are 10, 80 and 30 respectively for the three inputs (milk flow rate, hot water flow rate and power input). The arx322 model has Loss function of 0.0334283 and final prediction error (FPE) of 0.034922 .

\subsection{PID Controller}

The milk flow rate is set to constant, because our pasteurization is high temperature short time pasteurization that takes only 15 seconds. In order to meet this requirement we have to set our milk flow rate at $326 \mathrm{ml} / \mathrm{min}$. The hot water and the power input are let for PID controller (Figure 10) to adjust them until the set point $(720 \mathrm{C})$ is reached.
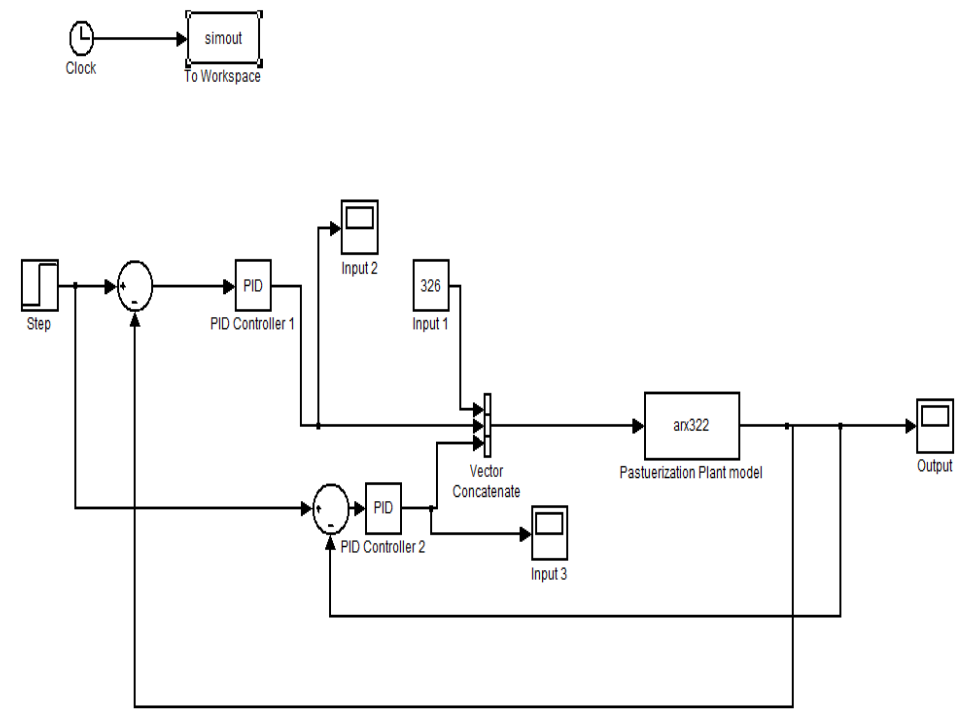

Figure 10. Simulink block diagram of PID controller

The two PID controllers tuned by trial and error method until the set point is tracked efficiently by using Simulink software. Table 1 is the setting of the two PID controllers to get best set point tracking.

Table 1. PID controller setting

\begin{tabular}{ccc}
\hline Setting & PID controller 1 & PID controller 2 \\
\hline $\mathrm{Kc}$ & 0.06 & 0.3 \\
$\mathrm{Ti}$ & 0.12 & 0.0009 \\
$\mathrm{Td}$ & 1 & 0.9 \\
\hline
\end{tabular}

Based on this setting the output temperature response is shown in Figure 11. 


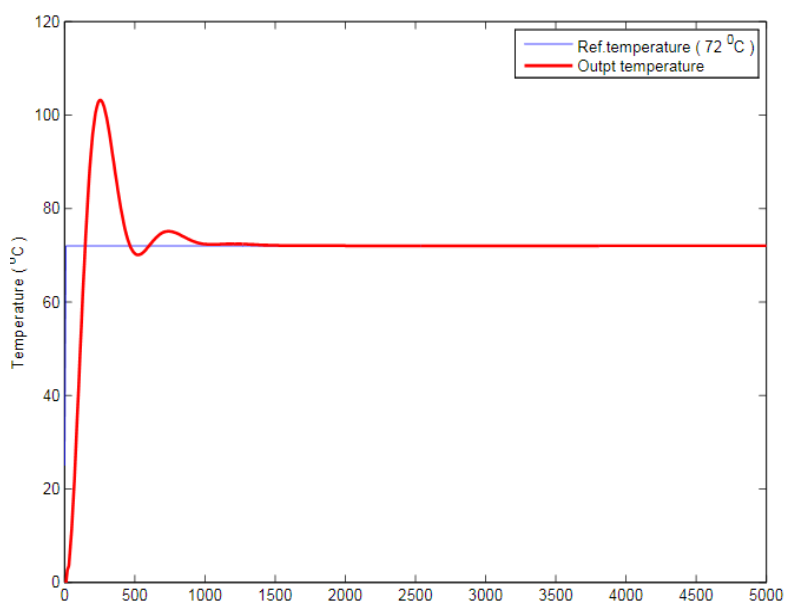

Figure 11. Output temperature (T1) response using PID

\subsection{MPC Controller}

The prediction horizon of the system should be large enough to cover the settling time as shown in Figure 12. The prediction horizon of 200 samples gives better settling than the others prediction horizon intervals as shown in Figure 13.
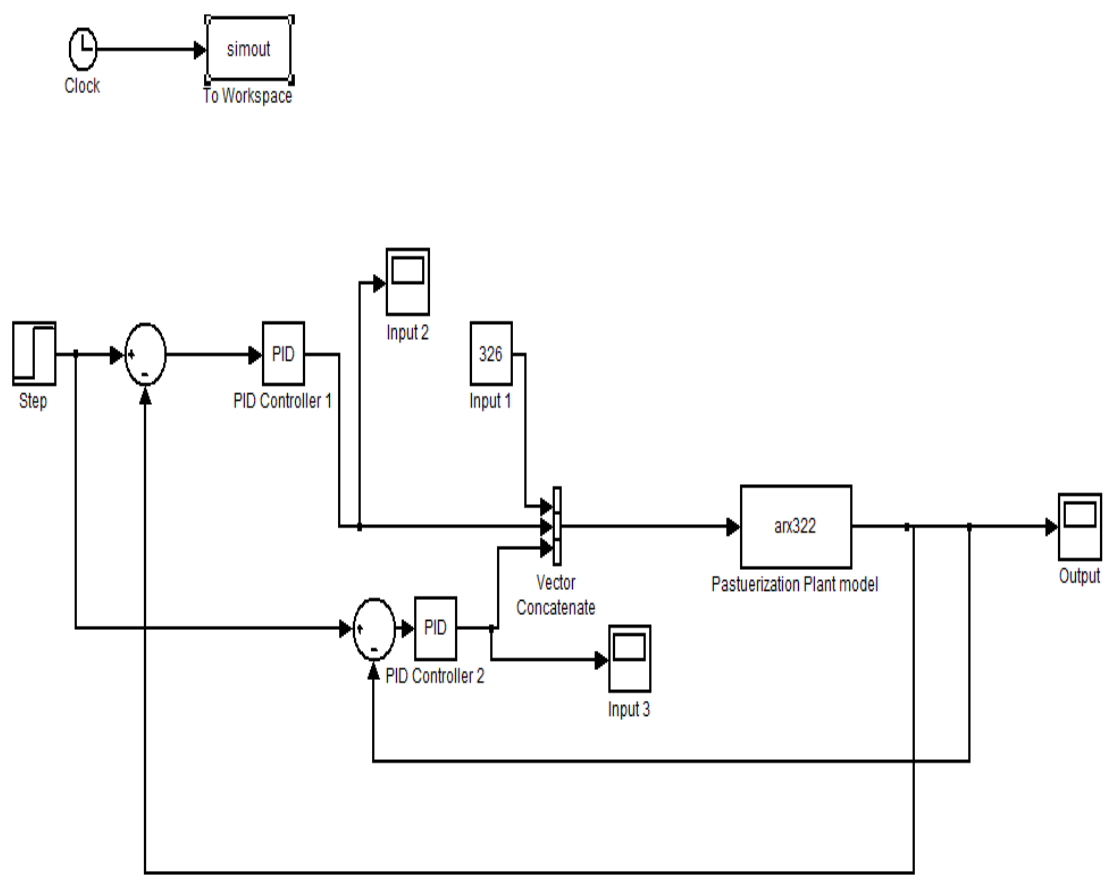

Figure 12. Simulink block diagram for MPC design 


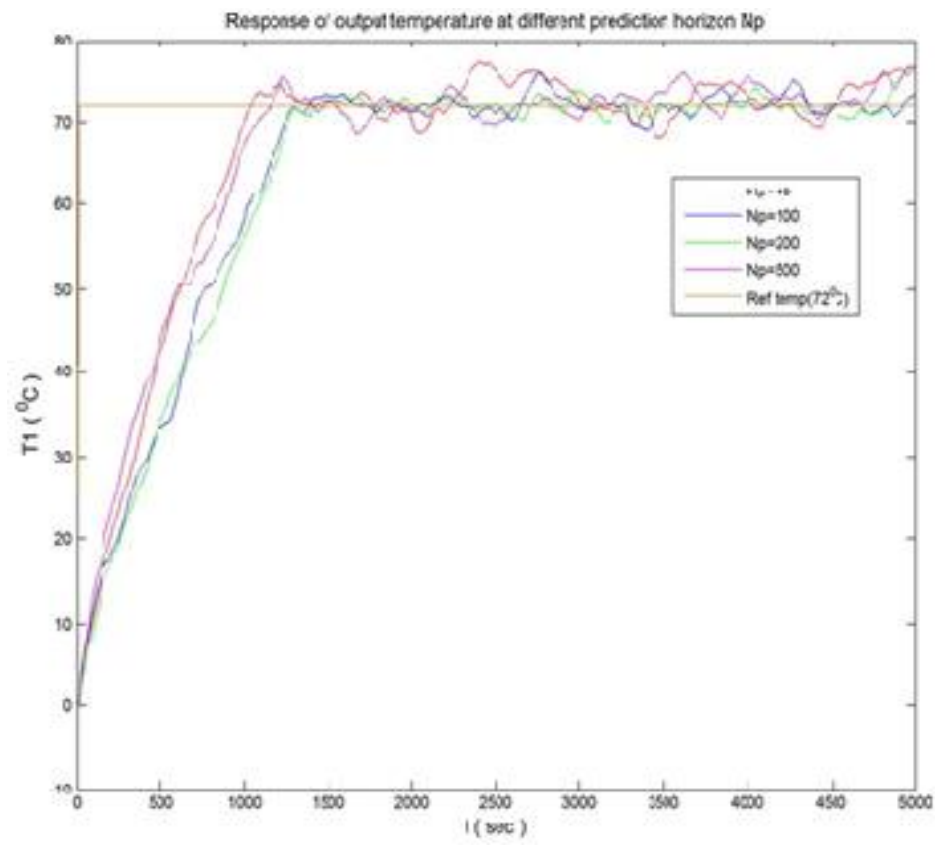

Figure 13. Prediction horizons tuning for MPC

The control horizon for different system should be different. It depends on the output signal of the system. In the most cases the control horizon should be large enough to get the reasonable stabilize output signal of the system. The long control horizon is required to improve the performance. Control interval of 95 is taken because beyond that it makes overshoot and becomes unstable, as shown in Figure 14.

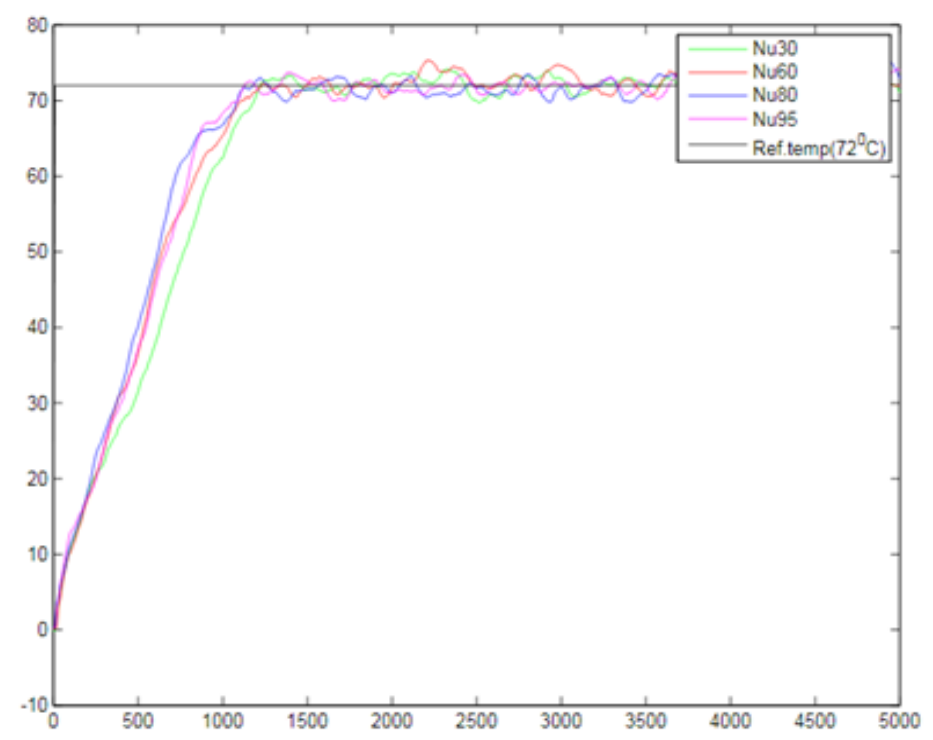

Figure 14. Controller horizon tunning for MPC

The output weight is 1 and input weight is zero. For the above values of prediction and control horizons, input and output weights and plant constraints, the optimized output temperature response using MPC is shown in Figure 15.

Bulletin of EEI Vol. 6, No. 1, March 2017 : 24 - 35 


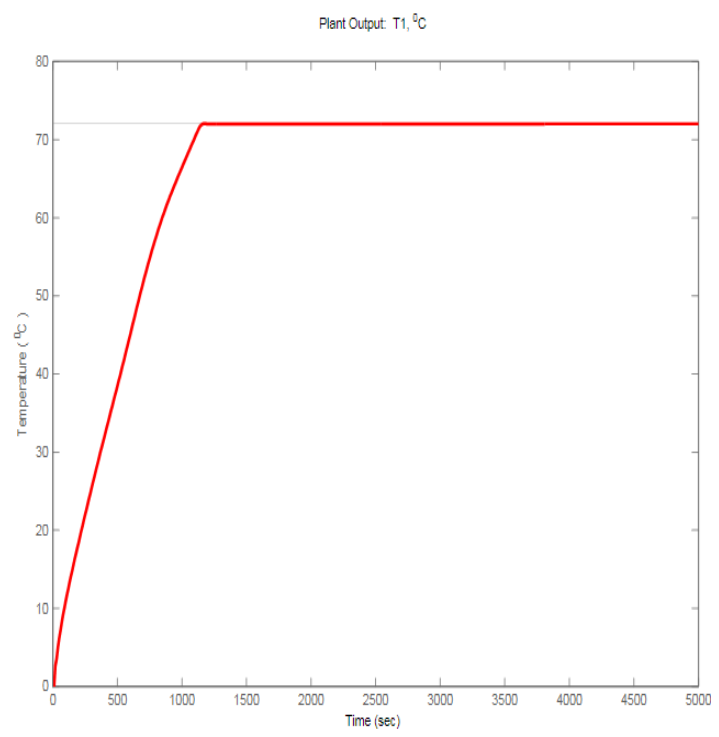

Figure 15. Output temperature response using MPC

\subsection{Stability Analysis of MPC}

The stability analysis of MPC can be checked using pole-zero location of the closed loop response after using MPC. All the poles and zeros are existed inside unit circle for the three inputs to one output relations as shown in Figure 16.
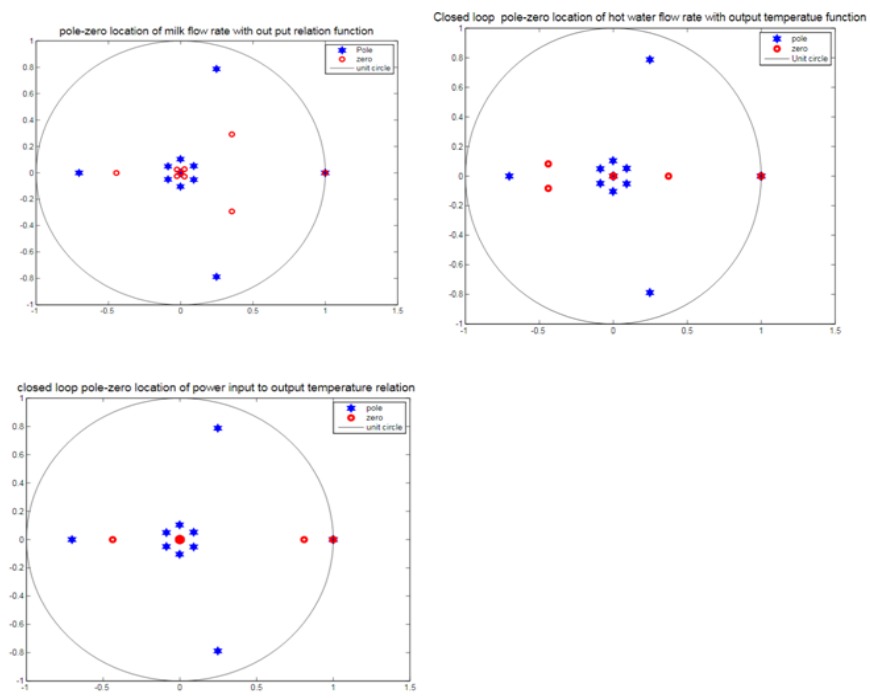

Figure 16. Closed loop pole-zero locations

\subsection{Comparison MPC and PID Controller}

The output temperature should be maintained at $720 \mathrm{C}$ which is the optimum temperature for high temperature short time (HTST) pasteurization process, but PID controller have overshot above this temperature, while MPC targets the set point exactly. The rise time of PID controller is better than MPC, but this may be due to high adjustment of power input out of the constraint region as shown in Figure 17. 


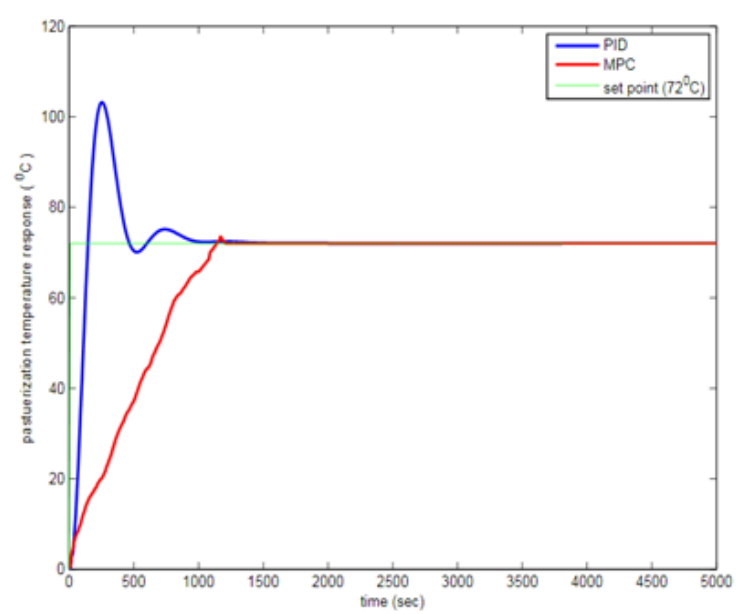

Figure 17. Output pasteurization temperature response

MPC can better set point tracking ability than PID controllers because this controller working in optimal condition by considering process constraints, that why there is no overshoot during MPC control action. Both controllers have the same settling time. MPC produces no overshoot while PID have higher overshoot.

\section{Conclusion}

From the above results and discussion we identified the best controller for pasteurization process to be MPC. Maintaining the temperature at a constant value is a critical issue in many of the Industries. MPC fulfills these type of difficulties by bringing the process variable to the desired set point as early as possible. MPC controller are more suitable or complex process control applications. MPC controller has $0 \%$ overshoot and minimum settling time as compared with PID controller that we used in this process. Consolidating the performance of all these responses MPC is the best controller for our pasteurization process.

\section{Acknowledgement}

The authors wish to thank Faculty of chemical and food engineering process control laboratory workers for their technical support and also Dr. Nigus Gabeye, Mr. Zewdu Tsegaye and Mr. Zelfikir Jemal for their comments and suggestions.

\section{References}

[1] Diederich Hinrichsen, Anthony J Pritchard. Mathematical Systems Theory I. Modeling, State Space Analysis, Stability and Robustness, 1.6: Heat Transfer. Springer Berlin Heidelberg New York: 2000: 70

[2] Negiz A, Cinar A, Schlesser JE, Ramanauskas P, Armstrong DJ, Stroup W. Automated control of high temperature short time pasteurization. Food Control 7.1996: 309-315

[3] Subhransu Padhee. Performance Evaluation of Different Conventional and intelligent. Sandviken, 80176 Gävle, Sweden.

[4] Orukpe PE. Basics of Model Predictive Control. Imperial College, London. April 14, 2005.

[5] ARMFIELD Process plant trainer (PCT23) Manual

[6] Lennart Ljung. System Identification: Theory for the user. Second Edition, Linköping University, Sweden, Prentice Hall PTR; 1999.

[7] Escobet, J Quevedo. Linear Model Identification Toolbox for Dynamic Systems. Department ESAll, Universitat Polittaka de Catalunya. Spain, IEEE. 1998.

[8] Astrom Karl J, Hagglund Tore. Advanced PID Control, Department of Automatic Control, Lund Institute of Technology. Lund University.

[9] Yuvraj Bhusan Khare, Yaduvir Singh. PID control of Heat Exchanger System. Punjab, India, 2010.

[10] Sidharta Dash, Mihir Narayan Mohanty. Analysis of Outliers in System Identification using WMS algorithm. India: 2012.

[11] J Rossiter. Model-based predictive control: A Practical Approach. CRC Press LLC: 2003. 
[12] MATLAB R2008b User Manual.

[13] F Yarman, BW Dickinson. Autoregredon Estimation Using Final Prediction Error. Princeton University, Princeton, NJ, Proceedings of the IEEE, Aug.1982; 70.

[14] Adriaan Van Den Bos. Parameter Estimation for Scientists and Engineers. Published by John Wiley \& Sons, Inc, Hoboken: New Jersey: 2007.

[15] Garcia CE, Prett DM, Morari M. Model Predictive Control: Theory and Practice - a Survey. Automatica, 1989; 25(3): 335-348.

[16] Antonio Balsemin. Applications Oriented Input design for MPC: An analysis of a Quadruple water tank process. KTH Electrical Engineering degree project, Stockholm, Sweden August 2012.

[17] Bequette B Wayne. Process Control: Modeling, Design and Simulation. Prentice Hall PTR. December 26, 2002: 487-511.

[18] EC Kerrigan, JM Maciejowski. Soft constraints and exact penalty functions in Model predictive control. In Proc. UKACC International Conference (Control 2000). 